\title{
Knowledge Management in Software Enterprise
}

\author{
Mrs. S. Mala ${ }^{1}$, Dr.K.Alagarsamy ${ }^{2}$ \\ ${ }^{I}$ Assistant Professor, Department of Computer Science, M K U College, Madurai. Tamilnadu, India \\ ${ }^{2}$ Professor, Department of Computer Applications, Madurai Kamaraj University, Madurai. Tamilnadu, India
}

Abstract: Knowledge management is expected to be integral part any software development and services companies. Knowledge has become an important capital for many organizations in the international competition. So knowledge management is gradually becoming the core competence and key of sustainable development for organizations. Many enterprises have already used concepts and methods of knowledge management for operation and achieved remarkable results. Based on the analysis of knowledge management system, a framework model for enterprise knowledge management is presented in this paper. For an enterprise, it is necessary to build this knowledge management system to share knowledge resources, provide scientific supports for decision-making, face fiercely competitive market, and so on.

Keywords: Knowledge Management, Software Enterprise, Knowledge Creation, Generation, Acquisition, Application, Distribution, Identification

\section{Introduction}

This paper is a study of knowledge management and how it is carried out in Software enterprise. This document tries to cover the study of knowledge management and various approaches undertaken in the organizations. The purpose of the current study is to highlight the ways in which technical and social factors which Knowledge management systems can help the organization and its customers.

\section{What Is Knowledge?}

Knowledge has many interpretations and having different meaning at different contexts. There are many scholars and try to provide us the definitions.

Knowledge is a "Process of Knowing". Someone with knowledge has the "Capacity to Act". Knowledge is dynamically created, and is intrinsically linked to people, and is the basis of all action. Knowledge is not data or information, and should not be viewed as an object that can be leveraged by simply investing in Information Technology to manage it.

The term "knowledge" is defined in the Oxford Dictionary and Thesaurus (Oxford Dictionary and Thesaurus, 1995.) as: "awareness or familiarity gained by experience (of a person, fact, or thing)", "persons range of information", "specific information; facts or intelligence about something", or "a theoretical or practical understanding of a subject". A more philosophical (and positivist) view of knowledge is to see it as "justified true belief". upon it." $[2]$

"Knowledge is of two kinds: we know a subject ourselves, or we know where we can find information

Data, Information, Knowledge and Wisdom

- Data - unorganized and unprocessed facts and figures

- Information - aggregation of data after processing for meaning and purpose

- Knowledge - derived from information based on understanding of the perceived importance/relevance to a problem area and can be used to draw meaningful conclusions

- Wisdom - the ability to make good decisions based on knowledge and experience

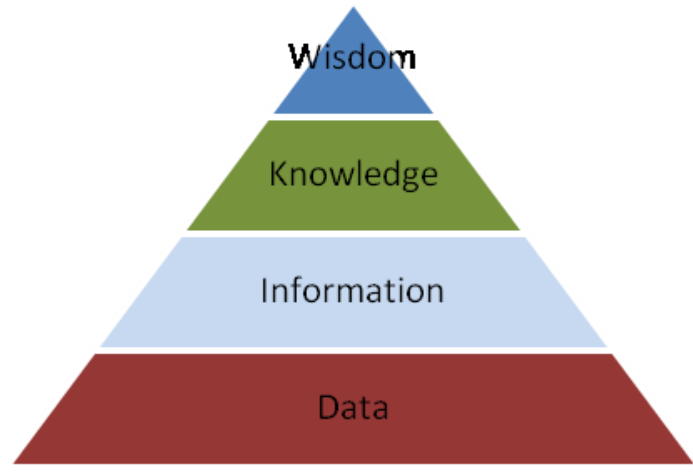




\section{WHAT IS KNOWLEDGE MEANS IN SOFTWARE ENGINEERING CONTEXT?}

Nowadays, knowledge about design and implementation models of current version of software system and also knowledge about application domain of software system are important for software engineering processes related to design, management, maintenance and modification of software system. Utilization of proper knowledge can streamline and speed up each phase of software system life cycle and increase quality of outcome software and length of its operation [6]

Software Engineering is concerned with building software systems which are large than would normally be tackled by a single individual, uses engineering principles in the development of these systems and is made up of both technical and non-technical aspects. [3]

The practical application of scientific knowledge in the design and construction of computer programs and the associated documentation required to develop, operate, and maintain them. [4]

The application of systematic, disciplined and quantifiable approach to the development, maintenance and operation of software; the application of engineering to software. [5]

\section{WHAT IS KNOWLEDGE WORK?}

Traditional management methods do not work for software because it is knowledge work. Drucker's definition of knowledge work is as follows.

- It involves concepts, ideas and designs.

- It is done in the workers' heads or on computers.

- The workers often know more about the work than their managers.

- Knowledge work cannot be tracked and managed by just watching it. Managing project knowledge work can make the difference between success and failure of large projects.

\section{TYPES OF KNOWLEDGE}

We often divide knowledge into two types, tacit and explicit knowledge [M. Polanyi, The Tacit Dimension, Doubleday, 1967, pp. 108.]. By tacit knowledge we mean knowledge that a human is not able to express explicitly, but is guiding the behavior of the human. For example how to ride a bike is something that is difficult to express, which you have to learn by trial and failure. Another example of tacit knowledge is the struggle of Japanese engineers to make a machine that bakes bread. According to Nonaka and Takeuchi [I. Nonaka and H. Takeuchi, The Knowledge-Creating Company, Oxford University Press, 1995, pp. 284], there were several trials to construct such a machine, but the bread simply did not taste as well as bread made by human bakers. The company NEC decided to send people to a local baker to see how the process of making bread was carried out. The researchers returned with new insight on the kneading process, and later were able to replicate this in their machine. This is an example of tacit knowledge that is difficult to transfer by other means than looking at someone who are actually baking bread.

Explicit knowledge is knowledge that we can represent, for example in reports, books, talks, or other formal or informal communication. So when we later talk about computer systems for knowledge management, it is only the explicit knowledge that can be managed in these kinds of systems; the tacit knowledge remains in the people! Some claim that tacit knowledge can be converted to explicit through externalization [I. Nonaka and H. Takeuchi, The Knowledge-Creating Company, Oxford University Press, 1995, pp. 284], and from explicit to tacit through internalization. We also find conversions from tacit to tacit - socialization, and explicit to explicit - combination.

Some terms related to knowledge, are experience and information. In normal English, experience means "actual observation of or practical acquaintance with facts or events", or "knowledge or skill resulting from this" [Oxford Dictionary and Thesaurus, 1995]. Most people see experience as a type of knowledge that you have gained from practice. Information is seen as "something told; knowledge", "items of knowledge; news". In normal English, it is difficult to distinguish the terms information and knowledge. Within artificial intelligence, information is often referred to as "data with meaning". The characters " $4 \mathrm{~m}$ " does not say much in itself, but if we know that "m" stands for "meters", it can be useful information. Knowledge is then often defined as information that is used (in an artificial intelligence-sense: in a computer system).

Nonaka and Takeuchi's (1995) theory on knowledge creation is based on the distinction between explicit and tacit knowledge.

- Explicit knowledge is knowledge that is transmittable in formal, systematic languages. It can be articulated in formal languages, including grammatical statements, mathematical expressions, specifications, manuals and so forth. It can be transmitted across individuals formally and easily.

- Tacit knowledge is personal and context-specific, and is therefore difficult to formalize and communicate. It is personal knowledge that is embedded in individual experience and involves intangible factors such as personal belief, perspective, and value system. Tacit knowledge is difficult to communicate and share in the organization and must thus be converted into words or forms of explicit knowledge. 


\begin{tabular}{|ll|cc|}
\hline \multicolumn{1}{|c|}{$[\mathbf{8}]$ Tacit Knowledge (Subjective) } & Explicit Knowledge (Objective) \\
\hline$\bullet$ & Knowledge of experience (no appropriate reasoning) & $\bullet$ & Knowledge of rationality (with justified reasoning) \\
$\bullet$ & Simultaneous knowledge (here and now) & $\bullet$ & Sequential knowledge (there and then) \\
$\bullet$ & Practice (analog knowledge) & $\bullet$ & Theory (digital knowledge) \\
\hline
\end{tabular}

The very idea in software engineering is to explicate knowledge in the forms of programs to be executed on computers. Software developers spend great effort developing programs, specifications, and models, while at the same time participating in close people-to-people interactions as members of software teams. According to Nonaka and Takeuchi organizational knowledge is created during the time the "conversion" between these forms takes place, i.e. from tacit to explicit and back again into tacit. Knowledge conversion is a "social" process between individuals and is not confined to one individual. Assuming that knowledge is created through interaction between tacit and explicit knowledge, four different modes of knowledge conversion are possible, see Figure below.

1.From tacit knowledge to tacit knowledge: Socialization

2. From tacit knowledge to explicit knowledge: Externalization

3. From explicit knowledge to explicit knowledge: Combination

4. From explicit knowledge to tacit knowledge: Internalization.

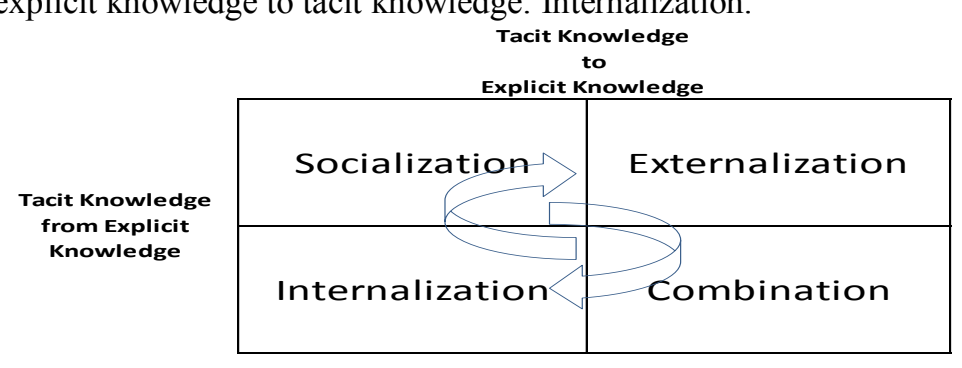

According to Nonaka and Takeuchi knowledge passes through different modes of conversion, which makes the knowledge more refined, and also spreads it across different layers in an organization.

\section{Knowledge Management in Software Engineering}

$\mathrm{KM}$ is simply about getting the right knowledge, to the right person, at the right time, in the right context. KM is a disciplined, systematic approach to leveraging people's expertise and information to improve organizational efficiency, responsiveness, competency, and innovation.

Software is playing an increasingly important and central role in all aspects of daily life-in government, banking and finance, education, transportation, entertainment, medicine, agriculture, and law. The number, size, and application domains of programs being developed have grown dramatically; as a result, billions of dollars are being spent on software development, and the livelihood and lives of millions directly depend on the effectiveness of this development.

There are many approaches to how software should be developed, which also affect how knowledge is managed. A main difference between methods here is if they are plan-based or traditional, which rely primarily on managing explicit knowledge, or agile methods, which primarily rely on managing tacit knowledge [S. Nerur, V. Balijepally, Theoretical reflections on agile development methodologies, Communications of the ACM 50 (2007) 79-83.]

A software engineering company who actively uses knowledge management is often referred to in the literature as a "learning software organizations". An organization that have to "create a culture that promotes continuous learning and fosters the exchange of experience" according to Feldmann and Althoff (2001). Another definition, by Dybå (2001) puts more emphasis on action: "A software organization that promoted improved actions through better knowledge and understanding". Edwards (2003) claims that knowledge management in software engineering is somewhat distanced from mainstream knowledge management, and claims the reason for this lack of "visibility" of software engineering in the wider knowledge management literature is a tendency for discussion of such topics to take place only at conferences for the software engineering community.

In software engineering, reusing life cycle experience, processes and products for software development is often referred to as having an "Experience Factory" [13]. In this framework, experience is collected from software development projects, and are packaged and stored in an experience base. By packing, we mean generalizing, tailoring, and formalizing experience so that it is easy to reuse. (V.R. Basili, G. Caldiera, H.D. Rombach, The experience factory, in: J.J.Marciniak (Ed.), Encyclopedia of Software Engineering, 1, John Wiley, New York, 1994, pp. 469-476.) 
The purpose of Knowledge Management is about how the organization can inspire, cultivate, and empower people to improve and share their "Capacity to Act". In so doing, KM should be viewed as a strategic issue for the whole organization as KM will help to steer it to become a knowledge-creating organization. A knowledge-creating organization will have the ability to innovate, develop new products, create new markets and respond quickly to competitions.

By better managing a company's knowledge base, some of the potential benefits are:

- Overcoming internal barriers to communicate and sharing;

- Enabling an innovative culture;

- Enhancing customer relationships;

- Increasing production outputs; and

- Developing more valuable IPR

KM must have a strong strategic and operational focus in order to succeed in achieving its business purpose. KM will require significant changes to "the way things are" in the organization in order to create a new environment for people to create, share and leverage knowledge.

Without top management involvement and support, KM will unlikely produce the intended results as fundamental shifts in strategic directions that are required might not be able to be implemented. Last but not least, $\mathrm{KM}$ is not about data and information management and exchange. So it will not help to invest heavily in IT software and support.

A company's business and work processes are carried out based on the policies and strategies under the company's missions and goals. Resources are committed to the projects and existing competencies are leveraged to perform the necessary activities. Without having to consciously knowing it, the company is utilizing its knowledge assets in its daily activities. But knowledge assets will not be efficiently used without proper establishment of a knowledge management system.

A knowledge management system allows the company to strategically leverage its knowledge assets to provide differentiations in its outputs from the business and work processes, and greater impacts which help to sustain or increase their competitive advantages. The following are the steps involved in the Knowledge management process.

\section{Knowledge Creation and Generation}

Companies create a great amount of data and information in their daily business activities. It would be essential for the company to have a system of managing the newly created information so it can be reused to solve new problems or leveraged to value-add to other business activities. For example, high technology companies may often receive a lot of feedbacks from customers on their products. This kind of information could be very useful for the R\&D team to come up with new improved products.

Companies may find that they cannot meet their knowledge requirement from their available knowledge assets. The gap will have to be filled either by internally developing new knowledge or acquiring the knowledge from external sources. Knowledge creation can only be achieved in a creative environment that encourages teamwork and the use of creative potential. If manage successfully, the process can expand or change the company's knowledge base to meet the company's current and future needs.

\section{Knowledge Acquisition}

Knowledge acquisition includes the elicitation, collection, analysis, modeling and validation of knowledge for knowledge engineering and knowledge management projects.

\section{Knowledge Identification \\ Knowledge Adaptation \\ Knowledge Organization}

This is a management idea, describing an organization in which people use systems and processes to generate, transform, manage, use, and transfer knowledge-based products and services to achieve organizational goals. A knowledge organization also links past, present, and future by capturing and preserving knowledge in the past, sharing and mobilizing knowledge today, and knowledge organizations can be viewed from a number of perspectives: their general nature, networks, behavior, human dimensions, communications, intelligence, functions, and services.

\section{Knowledge Distribution \\ Knowledge Codification}

Data and information need to be collected and analyzed in order to turn them into useful knowledge. This is the stage where tacit knowledge is converted into explicit knowledge and is very critical to the success of the other two stages - application and transfer. Without documenting and codifying tacit knowledge, its transfer for the purposes of learning and utilization, both internally and externally, will be difficult to achieve. Furthermore, legal protection of these valuable knowledge assets can only be done if the knowledge has been codified. For 
examples, patent applications require the complete disclosures of the inventions and trade secrets require the demonstration of safe-keeping of documented information. The legal rights come with IP protection offers the company a distinct advantage which can be used to derive revenues from IP licensing or exclusive rights to commercialize.

\section{KNOWLEDGE APPLICATION}

It is not unusual for companies to not to know how to generate value from the use of the knowledge assets they have. It is worse when a company does not even know the kind of knowledge it has. Knowledge Management offers a management system for the company to ensure that their knowledge assets when created are properly documented, and that the knowledge in different domain owners will be shared within the organization.

When knowledge assets are documented and shared, knowledge utilization will be facilitated. This is the stage in Knowledge Management where value creation is delivered. By harnessing knowledge from different knowledge domains and competencies across the organization, direct impacts to the missions and goals of the company can be achieved.

\section{KNOWLEDGE TRANSFER}

One of the advantages of knowledge is that knowledge is dynamic. Knowledge can be adapted and evolved through the processes of learning and sharing. The impact made by individual knowledge is not as great as collective knowledge so sharing within the organization should be encouraged.

When a company has limited capability to effectively use certain knowledge, it would be worthwhile to consider external transfer to third parties who may have the competencies to utilize the knowledge for value creation. For example, a company may have invented a new technology but it does not have the capability to produce products based on such invention. The technology can be licensed to a third party who has the production facilities and the marketing and sales capability to sell the new product. To ensure success of this technology transfer, it is essential that tacit knowledge and procedural knowledge are converted to explicit knowledge for easy learning, adaptation and utilization.

\section{MAPPING OF SOFTWARE ENGINEERING LIFECYCLE WITH KM PROCESSES}

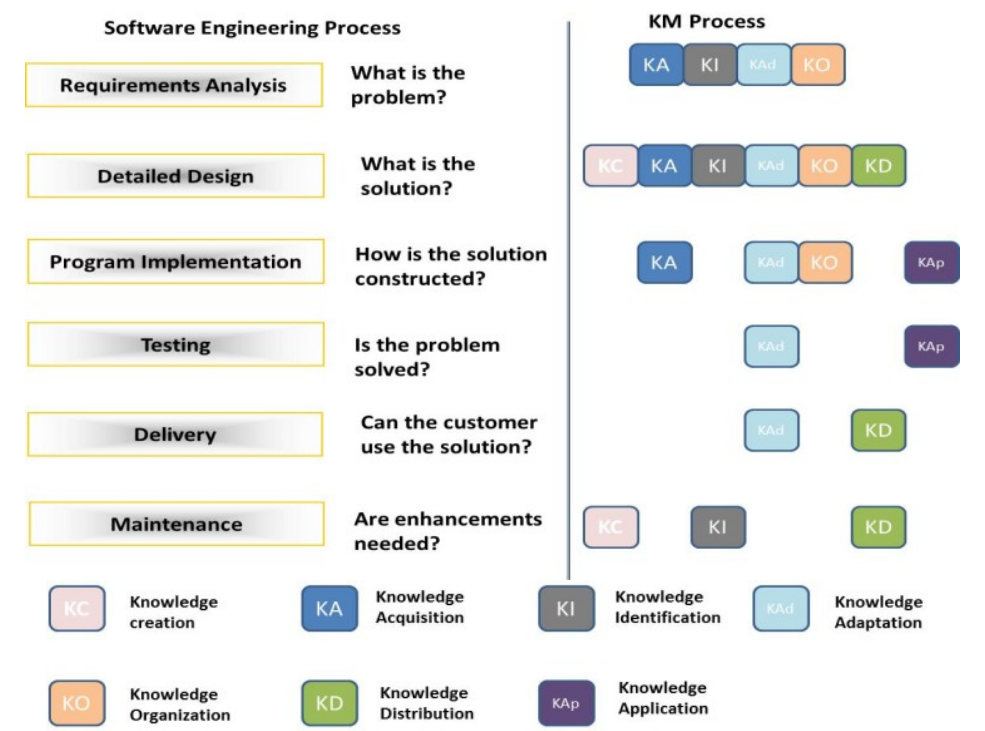

In Software engineering lifecycle, knowledge management applied in every single phase. There are many takeaways and learning's fed into the system for future application. The above diagram explains what knowledge management process applied in Software development lifecycle.

\section{KNOWLEDGE MANAGEMENT SYSTEMS}

Knowledge management (KM) is the management of explicit and tacit knowledge with data and information of the object domain to create value. Being in a knowledge-intense industry, good KM systems are important for the management of processes. 


\section{CONNECTING PEOPLE TO CONTENT}

This part of the KM consists of knowledge repositories that include processes for creation, distribution, and use of explicit knowledge. The Reusable Components Repository facilitates the use of previously developed components to reduce redundancy.

\section{CONNECTING PEOPLE TO PEOPLE}

This part helps in connecting people in need of knowledge to people with knowledge. Lists of experts in particular areas are maintained, and people seek information from these experts.

\section{BEST PRACTICES FOR PROJECTS}

This section contains the quantitative (metrics) and qualitative data for all the projects. Regardless of the claims about ambiguities surrounding the definition of knowledge, as well as the importance and necessity of KM, software processes continue to evolve. This is because software developers possess highly valuable knowledge relating to the product development, the software development process, project management and technology in general. As a knowledge-intensive work, the software development process involves both explicit and implicit knowledge. This knowledge is dynamic and evolves with technology, organizational culture and the changing needs of organization's software development practices.

There are cases where this knowledge is created at irregular intervals and the value of its use can only be displayed overtime. The use of the Internet facilitates the storage and utilization of activities, thus improving the quality of the software development process. Experience also plays a major role in knowledge-related activities. Software development can be improved by recognizing the related knowledge content and structure as well as the required appropriate knowledge, and performing planning activities.

This is how most of the software organization follows to implement their KM systems and monitor through periodic reviews.

\section{FUTURE WORK}

There are many aspects to look into with respect to applying Knowledge Management in Software enterprise. There is scope for research in this area about how the Knowledge Management practices followed in current software industry and analyze the trends to provide effective management system for the organization to follow. The following are the topics were further research can be done and arrive at some conclusions.

- Application of KM in software engineering Organizations

- Proposal of KM Framework

- Analysis of KM usage effectiveness

- Researching about KM from various SW development companies and analysis of KM usage

\section{Conclusion}

This paper discussed in detail about Knowledge Management and its application in Software Development lifecycle. It also discussed brief view about Knowledge, Knowledge Management and the process involved in knowledge management and mapping the KM process to Software Engineering Development lifecycle. This paper also outlined what further research can be done to arrive at effective knowledge management process for Software enterprise.

\section{References:}

[1]. Research on Knowledge Management System in Enterprise - Hua Jiang ; Sch. of Economic \& Manage., Hebei Univ. of Eng., Handan, China ; Cuiqing Liu ; Zhenxing Cui

[2]. Tacit knowledge - http://en.wikipedia.org/wiki/Tacit_knowledge

[3]. Explicit knowledge - http://en.wikipedia.org/wiki/Explicit_knowledge

[4]. Ref. Nonaka I., Takeuchi H., The Knowledge Creating Company, (1995), Oxford University Press

[5]. Knowledge in software life cycle - Havlice, Z.; Dept. of Computer\& Inf., Tech. Univ. of Kosice, Kosice ; Kunstar, J.; Adamuscinova, I. ; Plocica, O.

[6]. Nonaka, I. and Takeuchi, H. 1995. The Knowledge-Creating Company. Oxford University Press.

[7]. Dybå, T., Kitchenham, B. A. and Jørgensen, M. 2005. Evidence-Based Software Engineering for Practitioners. IEEE Software 22(1): 58-65.

[8]. http://www.k-strategian.com/knowledge-based-value-creation/

[9]. http://en.wikipedia.org/wiki/Knowledge organization_management)

[10]. http://www.epistemics.co.uk/Notes/40-0-0.htm

[11]. Nonaka and H. Takeuchi, The Knowledge-Creating Company, Oxford University Press, 1995, pp. 284

[12]. V.R. Basili, G. Caldiera, H.D. Rombach, The experience factory, in: J.J. Marciniak (Ed.), Encyclopedia of Software Engineering, 1, John Wiley, New York, 1994, pp. 469-476.

[13]. Oxford Dictionary and Thesaurus, 1995

[14]. M. Polanyi, The Tacit Dimension, Doubleday, 1967, pp. 108.

[15]. S. Nerur, V. Balijepally, Theoretical reflections on agile development methodologies, Communications of the ACM 50 (2007) $79-$ 83. 